\section{HÖHE DES BIP}

Das Bruttoinlandsprodukt (BIP) ist die Standardmessgröße des Werts der von einer Volkswirtschaft in einem bestimmten Zeitraum hergestellten Endprodukte und Dienstleistungen abzüglich des Werts der Importe. Das BIP ist zwar der wichtigste Indikator zur Erfassung der wirtschaftlichen Tätigkeit, sollte jedoch nicht als allumfassende Messgröße für das Wohlergehen einer Gesellschaft angesehen werden, da es verschiedene Aspekte des materiellen Lebensstandards der Bevölkerung unberücksichtigt lässt, ganz zu schweigen von anderen Aspekten, die die Lebensqualität der Menschen beeinflussen.

Das Pro-Kopf-BIP ist einer der Kernindikatoren für die Wirtschaftsleistung und wird häufig - trotz einiger bekannter Unzulänglichkeiten - als allgemeine Messgröße für den durchschnittlichen Lebensstandard oder wirtschaftlichen Wohlstand verwendet.

\section{Definition}

Was genau ist das Bruttoinlandsprodukt? „Brutto“ bedeutet, dass Abschreibungen für Ausrüstungen, Gebäude und sonstige im Produktionsprozess verwendete Investitionsgüter unberücksichtigt bleiben. „Inland“ bedeutet, dass es sich auf die Produktion bezieht, die auf dem Wirtschaftsgebiet des jeweiligen Landes erfolgt ist. Die Produkte beziehen sich auf Endprodukte und Dienstleistungen, die Gegenstand tatsächlicher oder unterstellter Käufe sind, als Endverbrauch der privaten Haushalte, der Haushalten dienenden privaten Organisationen ohne Erwerbszweck und des Staats sowie Anlageinvestitionen und Exporte (abzüglich Importe).

\section{Überblick}

Das Pro-Kopf-BIP lag 2010 im OECD-Raum insgesamt bei 33971 US-\$. Fünf OECD-Länder wiesen 2010 ein Pro-KopfBIP von deutlich mehr als 40000 US-\$ aus - Luxemburg, Norwegen, die Vereinigten Staaten, die Schweiz und in geringerem Maße auch die Niederlande. In vier OECDLändern - Australien, Dänemark, Irland und Österreich lag das Pro-Kopf-BIP im Jahr 2010 knapp über 40000 US-\$, während es sich in 13 Mitgliedsländern auf weniger als 30000 US-\$ belief, wobei sich die Türkei, Chile und Mexiko am unteren Ende der Verteilung befanden.

Im Jahr 2000 war das Pro-Kopf-BIP der Vereinigten Staaten 44\% höher als der OECD-Durchschnitt; im Jahr 2010 hatte sich die Differenz auf 37\% verringert. Japans Pro-Kopf-BIP fiel 2010 knapp unter den OECD-Durchschnitt, während es im Jahr 2000 noch knapp über dem OECD-Durchschnitt gelegen hatte.

Den stärksten Rückgang des Pro-Kopf-BIP im Verhältnis zum OECD-Durchschnitt verzeichneten zwischen 2000 und 2010 Israel, Island und Italien. Den größten Anstieg des relativen Pro-Kopf-BIP in diesem Zehnjahreszeitraum wiesen dagegen Luxemburg, die Slowakische Republik, Norwegen und Estland auf. Die Länder am unteren Ende der Verteilung (Chile, Mexiko und die Türkei) konnten das relative Niveau ihres Pro-Kopf-BIP im Verhältnis zum OECD-Durchschnitt ebenfalls verbessern.

\section{Vergleichbarkeit}

Alle Länder erfassen Daten nach dem System der Volkswirtschaftlichen Gesamtrechnungen von 1993 (SNA 1993), mit Ausnahme Australiens, wo die Daten nach dem neuen SNA 2008 erfasst werden. Dabei ist jedoch zu beachten, dass die Unterschiede zwischen SNA 2008 und SNA 1993 keinen wesentlichen Einfluss auf die Vergleichbarkeit der hier dargestellten Indikatoren haben, weshalb die Daten der einzelnen Länder gut miteinander vergleichbar sind.

Für einige Länder wurde das letzte Jahr der Zeitreihe vom Sekretariat geschätzt. Für jene Länder, die ihre Methodik geändert, aber nur für einige Jahre revidierte Daten geliefert haben, wurden auch Vergangenheitsdaten geschätzt.

Bei der Interpretation des Pro-Kopf-BIP ist Vorsicht geboten, da beispielsweise in Luxemburg und in geringerem Umfang auch in der Schweiz die Zahl der Grenzgänger relativ hoch ist. Solche Arbeitskräfte tragen zum BIP bei, werden jedoch nicht in den Einwohnerzahlen erfasst.

\section{Quelle}

- OECD (2012), National Accounts of OECD Countries, OECD Publishing.

- Für Brasilien und Indien: Internationaler Währungsfonds (IWF) (2009), World Economic Outlook, IWF, Washington DC.

\section{Weitere Informationen}

\section{Analysen}

- OECD (2012), OECD-Wirtschaftsausblick, OECD Publishing.

- OECD (2012), OECD Economic Surveys, OECD Publishing.

- OECD (2011), Towards Green Growth, OECD Publishing.

- OECD (2003), Die Quellen wirtschaftlichen Wachstums in den OECD-Ländern, OECD Publishing.

\section{Statistiken}

- OECD (2011), National Accounts at a Glance, OECD Publishing.

\section{Zur Methodik}

- OECD (2000), System of National Accounts, 1993 - Glossary, OECD Publishing.

- Vereinte Nationen, OECD, IWF und Eurostat (Hrsg.) (2010), System of National Accounts 2008, Vereinte Nationen, Genf.

\section{Online-Datenbanken}

- OECD National Accounts Statistics.

- OECD Economic Outlook: Statistics and Projections.

Websites

- OECD Economic Outlook - Sources and Methods, www.oecd.org/eco/sources-and-methods. 
Pro-Kopf-BIP

US-Dollar, jeweilige Preise und KKP

\begin{tabular}{|c|c|c|c|c|c|c|c|c|c|c|c|c|c|}
\hline & 1999 & 2000 & 2001 & 2002 & 2003 & 2004 & 2005 & 2006 & 2007 & 2008 & 2009 & 2010 & 2011 \\
\hline Australien & 26816 & 27968 & 29077 & 30314 & 31875 & 33306 & 34882 & 36814 & 38744 & 38964 & 39904 & 40790 & .. \\
\hline Belgien & 25366 & 27669 & 28524 & 30054 & 30292 & 31190 & 32204 & 34254 & 35667 & 37033 & 36744 & 37728 & 38711 \\
\hline Chile & 9088 & 9572 & 10004 & 10272 & 10784 & 11736 & 12690 & 13734 & 14628 & 15328 & 15201 & 16156 & 17312 \\
\hline Dänemark & 26926 & 28831 & 29432 & 30756 & 30430 & 32290 & 33196 & 36048 & 37723 & 39841 & 38303 & 40190 & 40929 \\
\hline Deutschland & 24994 & 25768 & 26707 & 27446 & 28354 & 29684 & 31117 & 33552 & 35559 & 37115 & 36052 & 37430 & 39187 \\
\hline Estland & 8752 & 9865 & 10691 & 11967 & 13371 & 14753 & 16531 & 19146 & 21583 & 22155 & 19791 & 20393 & 21938 \\
\hline Finnland & 23613 & 25674 & 26531 & 27531 & 27616 & 29863 & 30708 & 33140 & 36167 & 38080 & 35655 & 36307 & 37642 \\
\hline Frankreich & 23612 & 25249 & 26611 & 27676 & 27283 & 28185 & 29554 & 31426 & 33144 & 34167 & 33676 & 34256 & 35133 \\
\hline Griechenland & 16877 & 18249 & 19744 & 21401 & 22497 & 23861 & 24348 & 26803 & 27709 & 29569 & 29384 & 28444 & 26934 \\
\hline Irland & 26176 & 28932 & 30776 & 33274 & 34768 & 36796 & 38896 & 42522 & 45418 & 42575 & 39754 & 40478 & .. \\
\hline Island & 28632 & 28849 & 30438 & 31084 & 30776 & 33731 & 34992 & 35831 & 37171 & 39521 & 36666 & 35593 & 36084 \\
\hline Israel & 21333 & 23487 & 23400 & 23468 & 22195 & 23497 & 23256 & 23872 & 25449 & 25481 & 25479 & 26531 & .. \\
\hline Italien & 24345 & 25758 & 27276 & 26942 & 27271 & 27528 & 28280 & 30399 & 32056 & 33372 & 32250 & 31911 & 32939 \\
\hline Japan & 24600 & 25958 & 26567 & 27233 & 27966 & 29327 & 30443 & 31796 & 33370 & 33592 & 32119 & 33785 & .. \\
\hline Kanada & 27138 & 28485 & 29332 & 29911 & 31267 & 32837 & 35106 & 36863 & 38350 & 38985 & 37842 & 39050 & 40440 \\
\hline Korea & 15601 & 17197 & 18151 & 19656 & 20180 & 21624 & 22783 & 24247 & 26102 & 26689 & 26931 & 28797 & 30254 \\
\hline Luxemburg & 49072 & 53662 & 53923 & 57559 & 60728 & 64998 & 68372 & 78573 & 84559 & 89156 & 82981 & 86269 & 89801 \\
\hline Mexiko & 9259 & 10042 & 10134 & 10396 & 10882 & 11529 & 12461 & 13741 & 14486 & 15267 & 14343 & 15195 & .. \\
\hline Neuseeland & 20165 & 21036 & 22017 & 22775 & 23433 & 24498 & 25219 & 27020 & 28600 & 29077 & 29386 & 29711 & .. \\
\hline Niederlande & 26933 & 29414 & 30783 & 31943 & 31705 & 33197 & 35111 & 38088 & 40736 & 42929 & 41094 & 42196 & 42847 \\
\hline Norwegen & 29800 & 36137 & 37085 & 37052 & 38262 & 42479 & 47640 & 53846 & 55874 & 61332 & 54713 & 57259 & 61870 \\
\hline Österreich & 27186 & 28909 & 29025 & 30463 & 31319 & 32856 & 33637 & 36586 & 38073 & 39785 & 39026 & 40065 & 42132 \\
\hline Polen & 9996 & 10570 & 10948 & 11563 & 11986 & 13010 & 13786 & 15077 & 16759 & 18024 & 18926 & 19908 & .. \\
\hline Portugal & 16744 & 17797 & 18507 & 19146 & 19456 & 19854 & 21369 & 22967 & 24201 & 24939 & 24938 & 25444 & 25352 \\
\hline Schweden & 25976 & 27957 & 28226 & 29278 & 30420 & 32494 & 32701 & 35703 & 38478 & 39613 & 37339 & 39346 & 41348 \\
\hline Schweiz & 30626 & 32403 & 33062 & 34354 & 34245 & 35593 & 36648 & 40537 & 44362 & 47552 & 46343 & 48657 & .. \\
\hline Slowak. Rep. & 10407 & 10983 & 12069 & 12966 & 13599 & 14654 & 16175 & 18383 & 20876 & 23214 & 22583 & 23264 & 24018 \\
\hline Slowenien & 16707 & 17554 & 18438 & 19759 & 20516 & 22268 & 23472 & 25444 & 27218 & 29065 & 27153 & 26941 & 27402 \\
\hline Spanien & 19824 & 21314 & 22578 & 24068 & 24755 & 25956 & 27392 & 30406 & 32233 & 33130 & 32150 & 31904 & 32501 \\
\hline Tschech. Rep. & 14782 & 15549 & 16833 & 17578 & 18768 & 20081 & 21268 & 23268 & 25457 & 25872 & 25617 & 25258 & 26054 \\
\hline Türkei & 8171 & 9172 & 8612 & 8667 & 8791 & 10162 & 11391 & 12895 & 13894 & 15025 & 14443 & 15604 & .. \\
\hline Ungarn & 11059 & 11884 & 13394 & 14669 & 15344 & 16188 & 16975 & 18299 & 18933 & 20432 & 20157 & 20556 & 21547 \\
\hline Ver. Königreich & 24253 & 26072 & 27568 & 28884 & 29845 & 31766 & 32732 & 34999 & 35736 & 35882 & 34487 & 35687 & 35441 \\
\hline Ver. Staaten & 33298 & 35050 & 35866 & 36755 & 38128 & 40197 & 42414 & 44522 & 46227 & 46647 & 45087 & 46588 & .. \\
\hline EU27 & 20607 & 21912 & 23045 & 23968 & 24521 & 25707 & 26868 & 29070 & 30770 & 31976 & 31142 & 31784 & 32721 \\
\hline OECD & 23002 & 24404 & 25185 & 25958 & 26715 & 28135 & 29573 & 31517 & 33087 & 33882 & 32860 & 33971 & .. \\
\hline Brasilien & 6861 & 7204 & 7354 & 7560 & 7698 & 8231 & 8603 & 9166 & 9900 & 10528 & 10453 & 11239 & .. \\
\hline China & 2163 & 2378 & 2615 & 2881 & 3217 & 3614 & 4102 & 4749 & 5554 & 6189 & 6786 & 7519 & .. \\
\hline Indien & 1447 & 1518 & 1585 & 1657 & 1779 & 1942 & 2153 & 2402 & 2677 & 2862 & 3039 & 3339 & .. \\
\hline Indonesien & 2243 & 2441 & 2552 & 2674 & 2825 & 3005 & 3207 & 3449 & 3727 & 3987 & 4155 & 4394 & .. \\
\hline Russ. Föderation & 5895 & 6798 & 7336 & 8010 & 9231 & 10228 & 11826 & 14923 & 16729 & 20268 & 18892 & 19833 & .. \\
\hline Südafrika & 6322 & 6640 & 6897 & 7184 & 7478 & 8000 & 8654 & 9336 & 10049 & 10453 & 10238 & 10498 & .. \\
\hline
\end{tabular}

\section{Pro-Kopf-BIP}

US-Dollar, jeweilige Preise und KKP, 2011

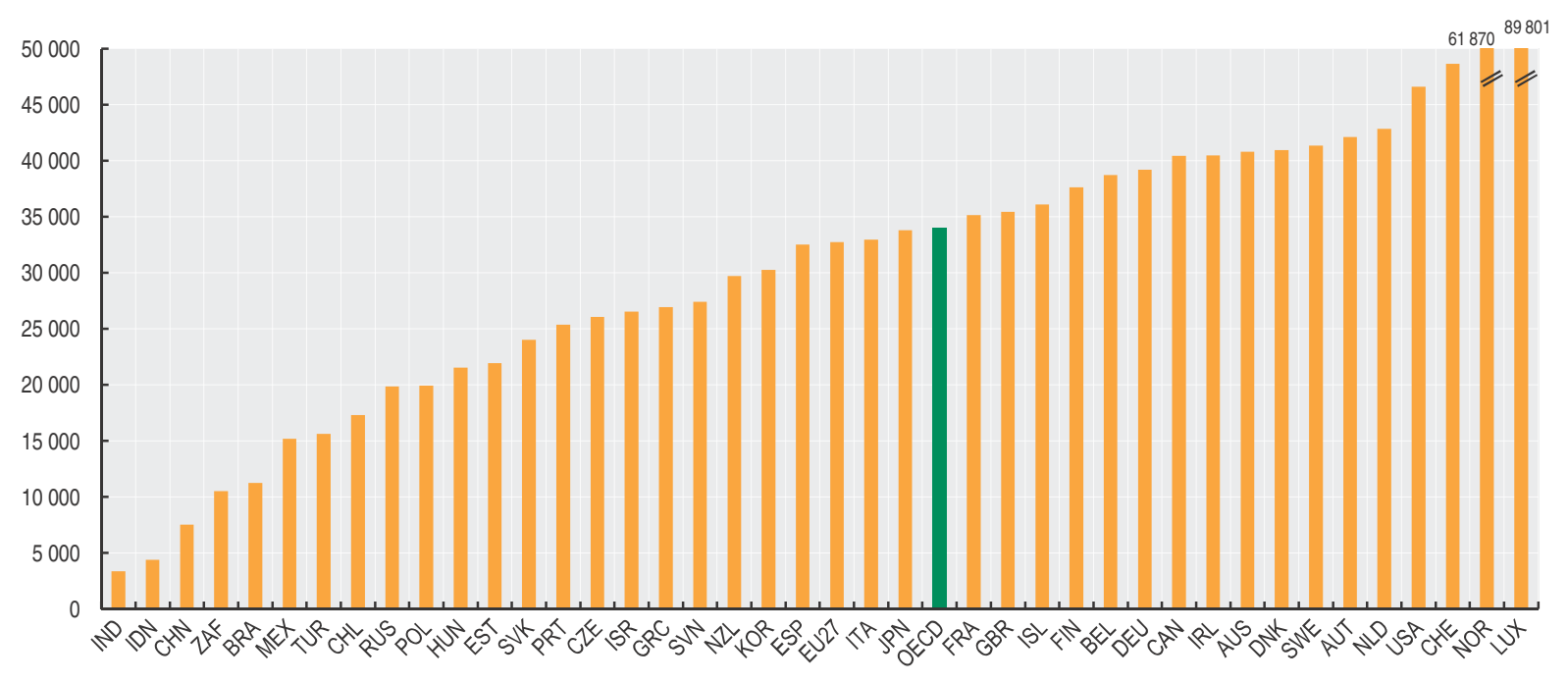




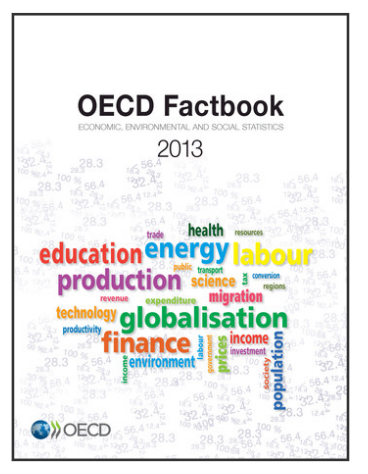

\section{From: \\ OECD Factbook 2013 \\ Economic, Environmental and Social Statistics}

Access the complete publication at:

https://doi.org/10.1787/factbook-2013-en

Please cite this chapter as:

OECD (2013), "Höhe des BIP", in OECD Factbook 2013: Economic, Environmental and Social Statistics, OECD Publishing, Paris.

DOI: https://doi.org/10.1787/factbook-2013-10-de

Das vorliegende Dokument wird unter der Verantwortung des Generalsekretärs der OECD veröffentlicht. Die darin zum Ausdruck gebrachten Meinungen und Argumente spiegeln nicht zwangsläufig die offizielle Einstellung der OECD-

Mitgliedstaaten wider.

This document and any map included herein are without prejudice to the status of or sovereignty over any territory, to the delimitation of international frontiers and boundaries and to the name of any territory, city or area.

You can copy, download or print OECD content for your own use, and you can include excerpts from OECD publications, databases and multimedia products in your own documents, presentations, blogs, websites and teaching materials, provided that suitable acknowledgment of OECD as source and copyright owner is given. All requests for public or commercial use and translation rights should be submitted to rights@oecd.org. Requests for permission to photocopy portions of this material for public or commercial use shall be addressed directly to the Copyright Clearance Center (CCC) at info@copyright.com or the Centre français d'exploitation du droit de copie (CFC) at contact@cfcopies.com. 